\title{
Author response to commentaries
}

\author{
Sara López Martín*
}

I want begin by acknowledging the fascinating and useful contributions these four renowned experts have made to my discussion paper; their comments and inputs honour me.

At the same time, and assuming, as Professor Henry Shue points out, that my proposal needs some clarification, I would like to address some of the issues raised by the discussion.

On the one hand, as Henry Shue and Önder Özkalıpcı mentioned, I would like to clarify that the dilemma was not related to therapeutic interventions, but only to forensic reports. Although we advocate for a retaining, as far as possible, of a therapeutic perspective in forensic work, I do not think that the same ethical principles that are applied in medical care can be applied here. We are not talking about the duty of care, but about challenges in forensic assessment, where the doctor and the psychologist play a completely different role. It is crucial to keep in mind here that the resources of small centres are limited.

To provide further detail:

1. The first confusion has to do with the assumption that we should apply the same

^) Lawyer, SiRa, Madrid.

Correspondence to: juridico@redsira.net approach to therapeutic and forensic work. This issue is not trivial because we think as Henry Shue has rightly pointed out that, from a clinical point of view, "giving therapy and composing a report for an asylum application are separable". Our centre, in fact, seldom provides "medical care", in the sense that Juliet Cohen and Önder Özkalıpcı describe it, and on these occasions, we have no doubts regarding the duty of care contained in the Hippocratic Oath ${ }^{1}$. The dilemma discussed relates to a different scenario.

2. As our rehabilitation professionals are from different professional backgrounds, and do not work from the same perspective, it is valuable to harmonize these approaches. We are a multidisciplinary team. Thus, from an ethical point of view, this deontologically-bounded activity is not restricted but instead extended (or doubly restricted), by different deontological provisions. Hence,

1 We provide therapeutic support, pharmacological treatment, sometimes separately, sometimes in combination; corporal therapy, medical forensic reports; medical-psychological-psychiatric forensic reports; legal advice, various methods of traditional healing when considered pertinent, among many other things. 
there are other concerns to be addressed in addition to the "duty of care". Furthermore, this may mean, as Cohen fears, having to make inroads beyond the boundaries of our medical ethics, which do not necessarily resolve all dilemmas.

As already stated, it is clear that the primary victim to be protected is the one who in front of us (Elizabeth Lira). However, here in this case, I would apply the logic of a single code of conduct, medical deontology, to cases that generate dilemmas for us. In that case, the big question is: once this victim is protected, does my commitment against torture cease? Am I not obliged to think that if, by helping, I indirectly facilitate torture of other people, or that, if they were tortured in the past, they have no reparation? I believe that a view that only contemplates a master key (the use of medical ethics for any situation) does not allow us to analyse the mediumand long-term consequences of what we do. Such a narrow view would not allow us to be accountable, to give explanations, to the potential victims of the person we are assessing, in the past or the future, if any at all.

In addition to this, other complexities move us away from prescriptions proposed surrounding the duty of care:

1. As we are not judicial forensic experts, but experts on behalf of the parties, we are not obliged to accept every case. Thus, we cannot rely on a commitment to accept all cases, in order to solve the dilemma.

2. This element also means that, when we accept a case, we make a "certain" commitment not to harm our client. What is more, we do not have "a duty to the Court to include in the report all relevant information", as Dr Cohen states, mainly when this may condition a refusal of asylum, with all the associated risks.

3. Indeed, our reports are not conclusive (Onder Özkalipc1 and Henry Shue), but often have a substantial weight in decision making, especially in less documented cases. I have to acknowledge that, on issues such as non-refoulement, I find it difficult to discern the boundaries between "not helping" and "harming" (Shue), precisely on account of the implications that the absence of help has in some cases.

4. In any case, our position on torture and the protection of victims is strong. We are simply in a particular situation, different from official forensic practice, but also different from a limited medical approach so that traditional anchors do not always allow us to face the challenges posed by such dilemmas.

I also have to disagree with statements about what it should be to "do our job well". I defend the importance of studying not only the general but also the specific contexts of the facts that affect our client's history. We are professionals who must rigorously analyse the facts (and I think this has little to do with judging the work of prison doctors or refusing to treat criminals or people we dislike). I find it hard to imagine our work without this "preliminary stage of inquiry", precisely because we analyse the consistency of symptoms and facts. This is not just related to medical sequelae, but also to psychological and psychiatric impacts, which are different, much more complex, and require specific procedures and methods that may go far beyond the analysis of external scars. We do not need much information for a medical report. However, we need it when we need to assess consistency with psychological symptoms or to justify the 
absence of psychological symptoms, which is what most often happens.

$\underline{\text { I also believe that this analysis affects trust, }}$ and this is not a problem that disappears with the acceptance of the case. Furthermore, distrust can increase as we know more about the case. This dynamic process is part of the work of making a judgement of consistency, and it is part of the work of the forensic expert. In any case, Elizabeth Lira's proposal of a case-by-case analysis seems not only inevitable but probably the only clear way forward at this point.

\section{Wrapping up and proposal}

Thus, grateful for the contributions and the possibility of thinking in greater depth about commentators' suggestions, below is a finetuning of my initial proposal, although significant changes are not incorporated.

These are our new proposed criteria:

1. Reject potential clients where there is conclusive evidence that they might be active perpetrators.

There is rarely conclusive evidence about the activities of the assessed person (except when that person flaunts victims in front of our horrified ears, and this has happened to us). We must offer an alternative. For example, to offer a clinical impact record instead of making a full Istanbul Protocol report.

In any case, the debate over whether or not to reveal suspicions to the victim is not resolved. It demands a risk-benefit analysis in a case-by-case study.

2. Reject potential clients that are sought by a national or international court for human rights violations.

Again, this will rarely happen. If there were to be injunctions, they would probably be from domestic national courts, of which we may not be aware, and, in any case, we have no reason to question a priori their ability to make a legitimate and safe prosecution. Once again, only on a case-by-case analysis would we know if we are dealing with a potential perpetrator of crimes against humanity and this in itself would help us to decide what to do (or not do!).

3. Not contribute to the refoulement of anyone - perpetrator or not- to a country that will not guarantee their physical and psychological integrity.

I have no doubts about this criterion. The only concern is that, because of the type of cases we usually see in asylum applications, this premise is always fulfilled and makes this debate sterile. I understand, however, that the debate about what kind of support we can offer depends on whether the case is sufficiently documented or on the weight that our report may have, among other factors that will determine whether not helping is or is not harmful.

Table 1. New proposed criteria

1. Reject potential clients where there is a conclusive evidence that they might be active perpetrators (define "conclusive evidence" and where to find it).

2. Reject potential clients that are sought by a national or international Court for human rights violations (how to discover whether this is relevant and actions to take in this case).

3. Not contribute to the refoulement of anyone-perpetrator or not- to a country that will not guarantee their physical and psychological integrity (decide what we do to help, provided that failure to do so will result in the possibility of return to their country of origin).

The discussion remains open... 